\title{
The Leap of Mind of Linguistic Humans over Animals
}

\author{
Zi-Jian Cai \\ CaiFortune Consulting, Suzhou, China \\ Email: hrsh8@126.com
}

How to cite this paper: Cai, Z.-J. (2018) The Leap of Mind of Linguistic Humans over Animals. Open Access Library Journal, 5: e4771.

https://doi.org/10.4236/oalib.1104771

Received: July 11, 2018

Accepted: August 6, 2018

Published: August 9, 2018

Copyright @ 2018 by author and Open Access Library Inc.

This work is licensed under the Creative Commons Attribution International License (CC BY 4.0).

http://creativecommons.org/licenses/by/4.0/

\begin{abstract}
Recently, it was suggested by Cai a new semantic/syntactic/episodic model of language, encompassing the sentential meanings to the neural linguistic components, and making it possible to install the whole linguistic functions into robots through the three semantic/syntactic/episodic components. In this article, it is considered some improvements of language brought to the brain and mind of humans over animals, and accordingly some of their impacts on the artificial intelligence. It is pointed out that language is the unique advantage of human brain over animal brain, especially efficient for mutual communication, situation summarization, procedural depiction, story narration, logic thinking, comprehensive integration, and so on. It is advantageous to symbolize or summarize the complex situations, and then to delineate or depict their successions by paragraphs and stories from sentences. Even though thinking requires additional memory, inference and assumption difficult for zip of computer, it can instead benefit from the assistance of language because it as well falls into situational or successional. In application, with the comprehension and expression of language corresponding to the animation and depiction in artificial intelligence respectively, it is possible for the artificial intelligence and adaptive robots to acquire the advantage of linguistic human brain over the animal brain, improving the depiction and communication of complex situations and successions, as well as learning the various complex skills and procedures through language. It is also reversely demonstrated the advantage of linguistic human brain over animal brain with the perspectives of language to greatly improve the robots.
\end{abstract}

\section{Subject Areas}

Linguistics, Psychology, Neuroscience, Artificial Intelligence, Computational Robotics 


\section{Keywords}

Semantic/Syntactic/Episodic Model of Language, Situation Summarization, Procedural Depiction, Thinking, Communication, Artificial Intelligence, Adaptive Robotics

\section{Introduction}

Up to now, native language is the high and complex mental activity unique to humans for communication and meditation, making the humans more superior than animals in mutual communication, situation summarization, procedural depiction, story narration, logic thinking, comprehensive integration, and so on. Recently, Cai extended the present declarative/procedural model underlying two kinds of neural components to a new semantic/syntactic/episodic model of three kinds of neural components, able to encompass the sentential meanings to the neural linguistic components [1] [2].

One of the most important applications for demonstration of neural components of language in brain lies in the promotion of artificial intelligence to assimilate and use the linguistic components as that in human brain. The animation and depiction of language in artificial intelligence correspond to the comprehension and expression of language in humans respectively. With the new neural linguistic model of three semantic/syntactic/episodic components able to encompass the sentential meanings and install the whole three semantic/syntactic/episodic components into the robots [1] [2], it is possible to make the artificial intelligence and adaptive robots acquire the advantage of linguistic human brain over the animal brain, greatly improving the animation, depiction and communication of complex situations and successions.

In this article, it is attempted to emphasize the advantage of linguistic human brain over the animal brain, and then to consider the consequential improvements in some important aspects of artificial intelligence and robots.

\section{Theoretical Breakthrough: The Three Semantic/Syntactic/Episodic Neurolinguistic Components of Brain}

Recently, Cai [1] [2] extended the present declarative/procedural model of Ullman and Pinker underlying two kinds of linguistic components [1] [2] [3] [4] [5] to a new semantic/syntactic/episodic model of three kinds of linguistic components in brain [1] [2], able to encompass the sentential meanings to the linguistic components that the declarative/procedural model of Ullman and Pinker has neglected [1] [2] [3] [4] [5].

It is known that memory can be divided into declarative memory for facts or episodes, and procedural memory for skills or habits [6] [7] [8]. According to Pinker, Ullman and so on [1] [2] [3] [4] [5], word belongs to the declarative 
memory while grammar belongs to the procedural memory. Whereas, declarative memory can further be divided into short-term memory and long-term memory. Short-term memory is the memory of precedent episodes or facts within minutes or seconds, while long-term memory is the memory of them more than hours or days ago [6] [7] [8]. Besides, remote retrograde memory is the long term memory lasting for decades of years [6] [7] [8]. Common words are acquired as remote retrograde memories from childhood [1] [2], just one kind of stable declarative memories.

Pinker, Ullman and so on integrated a declarative/procedural model for linguistic processing in brain. They suggested that the linguistic lexicon of word-specific knowledge should subserve the storage of meanings of words and phrases, and depend on the temporal-lobe substrates of declarative memory for the storage and usage of facts and events [1] [2] [3] [4] [5], while the linguistic grammar should subserve the sequential combination of lexical items by procedural rules into complex sentences, and depend on a network of specific frontal, parietal, basal-ganglia and cerebellar structures of procedural memory in the brain [1] [2] [3] [4] [5]. There are many evidences in support of this declarative/procedural model of language [1] [2] [3] [4] [5].

To extend this declarative/procedural model to a new semantic/syntactic/episodic model of three linguistic components in brain, Cai added that, besides the declarative/procedural components of language, the episodic coordination of cortical modality by reticular formations and nonspecific thalamic projections was also required for language performance and organization in addition [1] [2]. Many neurobiological evidences supported this suggestion [1] [2], as in the followings: 1) The reticular dopaminergic system may be involved in the linguistic modality organization, as the dopaminergic antagonists [9] [10] alleviate while the dopaminergic genes [11] phenotype the stuttering. 2) From the drugs in many assisting therapies of aphasia, it is implicated that the ascending reticular cholinergic projections, noradrenergic projections and nonspecific projections from intralaminar thalamic nuclei may participate in episodic coordination of cortical modalities in linguistic processes [12] [13]. 3) It is evidenced by numerous reports that the gamma bands of high frequency, subjected to modulation by the reticular cholinergic system [14] [15], associate with the word congruency at sentential level [16] [17] [18], and the episodic coordination of cortical modalities.

Now that it is well evidenced for the episodic coordination of cortical modalities at the sentential level, it is reasonable to further consider it for narration of paragraph and story. Complex paragraphs and stories certainly require more episodic coordination of cortical modalities. Language disorders occur in narrative discourse skills in patients with various pathologies [19] [20] [21], demonstrating diversity in cortical modalities. Besides, stuttering is also a disorder of discourse sometimes, as narrative therapy is auxiliary to treatment of stuttering [22] [23]. These facts implicate that episodic modality coordination of cortices is 
also required at high level during narration of paragraph and story.

In summary, herein it is briefly demonstrated that there are three semantic/syntactic/episodic linguistic components present in the human brain. With these semantic/syntactic/episodic neural components of language, it is possible to: 1) comprehend the various meanings of words and phrases; 2 ) organize the words and phrases by grammar into sentence; 3 ) determine one correct meaning of some words of multiple meanings by matching them with episodic associations with others in sentence and paragraph [24] [25]. Obviously, with these semantic/syntactic/episodic neural components of language, it is possible to animate and depict all linguistic processes with artificial intelligence.

\section{The Advantages of Human Brain with Language over Animal Brain}

\subsection{The Advantages of Language of Human Brain}

Native language is the high and complex mental activity unique to humans for communication and meditation, making the humans more superior than animals in mutual communication, situation summarization, procedural depiction, story narration, logic thinking, comprehensive integration, and so on.

The language is especially advantageous to symbolize or summarize the complex situations, and then to delineate or depict their successions. With the semantic/syntactic/episodic components of language [1] [2], the comprehension and expression of various meanings of words and phrases make the human brain able to symbolize, abstract, summarize and memorize the various characters and situations. The common procedural syntactic grammar of language [1] [2] makes the human brain easy to share, communicate and understand the language with common grammar among the regional ethnic society.

The language is more complex than simple sentences because there are many long sentences and clauses, and various in meanings. With several or many sentences, there can further form the various stories. It is necessary to adopt the episodic coordination to understand the meaning of the whole long sentences [1] [2]. Certainly, it is also necessary for narration of paragraph and story to use episodic coordination [19] [20] [21]. In computer simulation, classifying the sentence/paragraph into the episodic category of natural/social subjects like physics, biology, art, economy, and so on is designed to improve the comprehension of language [25]. Therefore, by forming the paragraph and story from sentences, it is able to comprehend, depict and communicate the complex situations and their successions by language, as well as to learn the various complex skills and procedures through language.

\subsection{The Brain Processing of Linguistic and Nonlinguistic Information}

With the semantic/syntactic/episodic components of language, the following brain structures are adopted: 1) Word comprehension is processed in the vari- 
ous cortices for remote declarative memory storage [1] [2] [4] [5]. 2) Grammatical rule is processed in the frontal, parietal, basal-ganglia and cerebellar structures of procedural memory in brain [1] [2] [4] [5]. 3) Episodic coordination of linguistic cortices is accomplished by the ascending reticular dopaminergic, cholinergic, noradrenergic systems and nonspecific thalamic projections [1] [2].

With the advantages of language easily and clearly manifested by the semantic/syntactic/episodic components, it is still necessary to further inspect the brain processing of nonlinguistic information. Beyond the structures for processing language, in brain there are only the limbic structures responsible for processing of emotions [6] [7] [26] [27], and right hemisphere responsible for processing of information other than language in healthy subjects [28] [29], such as the spatial and emotional information, including the prosodic information [2] [28].

The complex situations and their successions as processes can be present as both linguistic and nonlinguistic forms. Whereas, they are all processed along with language in humans by association with sentential or paragraphic contents in meanings. In this way, through the advantages of language, the human brain is able to depict and communicate the complex situations and their successions by language, as well as acquire and master the various complex skills and procedures through language.

The thinking of human brain requires the further addition of memory, inference and assumption difficult for zip of computer, which can instead fall into situational and successional. Accordingly, the human brain is also able to further think via the additional memory, inference and assumption by verbal and nonverbal components. Whereas, because most thinking is either situational or successional, language is likewise indispensable for assistance to the human thinking due to the same advantages as stated in Section 3.1, advantageous in dealing with the complex situational or successional thinking for the linguistic human brain over animal brain.

In all, with the semantic/syntactic/episodic components being formulated for language in human brain, the human brain has evolved the leaping advantages over the animal brain in depicting and learning the complex situations and their successions as processes.

\section{The Impacts on Artificial Intelligence from the Linguistic Advantages and Semantic/Syntactic/Episodic Components}

\subsection{The Feasibility of Semantic/Syntactic/Episodic Linguistic Components to Be Applied Fully Functional in Artificial Intelligence}

One of the most important advantages of the semantic/syntactic/episodic linguistic components over others lies in their feasibility to be applied in full functions to automated machines, making the artificial intelligence acquire the full linguistic ability as humans [1] [2] [24] [25].

According to the three components of the semantic/syntactic/episodic linguis- 
tic model [1] [2], Cai recently derived three principles to direct the machine translation respectively [24]. First, it was necessary to establish the dictionary for translation of words and phrases. Second, it was also necessary to read out the grammar of language to be translated from and to comply with the grammar of language to be translated into, arranging such parts of sentence as noun, verb and adjective into order. Third, it was in further necessary to determine one correct meaning for some words of multiple meanings by matching them with the episodic and statistical associations with others.

Besides, Cai recently discovered three types of episodic associations [25] prospective in applications to statistical translation, as: 1) Classifying the living/natural words and phrases of multiple meanings by behavior, adopting both the zoological/organizational/physical/categorical and affective/behavioral/logic/ characteristic/changing characters to classify the nouns and verbs, and so on, helpful to discern the meanings of them using these episodic associations with others within the sentence. 2) Likewise, classifying the sentence/paragraph into the category of natural/social subjects like physics, biology, art, economy, and so on. 3) Collecting the frequent word-pairs during statistical translation, such as "bank money", "war declaration", etc., helpful to determine the episodic associations of some prepositions or terminal "which" clauses. Besides, it was further suggested to use word episodic symbolization to symbolize all of the special characteristics and episodic associations of a word and then store them altogether with the word in computer easily [25].

The linguistic robots of artificial intelligence are more complex than machine translation. It is certainly necessary to install language functions into robots and make them more competent than translation machines. Likewise, first it is necessary to establish the dictionary for explanation of words and phrases [24]. Second, it is also necessary to install the grammar of language, arranging such parts of sentence as noun, verb and adjective into order [24]. Third, it is in further necessary to determine one correct meaning of some words of multiple meanings by matching them with the episodic and statistical associations with others [24], and even using the above mentioned three types of episodic associations [25]. As the robots need learning, the Bayesian Rule is usually adopted for the robots [24]. In this regard, the linguistic robots can acquire the full functions of language via the three semantic/syntactic/episodic components.

Especially, word episodic symbolization can symbolize both the special characteristics and episodic associations of a word and then store them altogether with the word in linguistic robots easily [25]. By this way, much nonverbal information, such as the spatial and emotional information, can be stored in association with words in linguistic robots, greatly increasing the ability of linguistic robots.

\subsection{The Advantages of Linguistic Robots over Animal Brain}

The animation and depiction in artificial intelligence corresponds to the comprehension and expression of language in humans respectively. Likewise, just as 
the humans, the robots with the acquisition of language would be greatly improved in mutual communication, situation summarization, procedural depiction, story narration, logic thinking, comprehensive integration, and so on.

The linguistic robots are also able to symbolize or summarize the complex situations, and then to delineate or depict their successions by paragraphs and stories from sentences. Likewise, with the semantic/syntactic/episodic components of language [1] [2], the comprehension and expression of various meanings of words and phrases would make the linguistic robots able to symbolize, abstract, summarize and memorize the various characters and situations. The common procedural syntactic grammar of language [1] [2] makes the linguistic robots easy to share, communicate and understand the language with the same grammar of language among the regional ethnic society, including those from both humans and robots.

The language is sometimes more complex with many long sentences and clauses, and various in meanings. With several or many sentences, there can further form the various paragraphs and stories. It is necessary for the linguistic robots to adopt the episodic coordination to understand the meaning of the whole long sentences [1] [2], and in further the paragraphs and stories from the sentences via categorical classification of subjects [25]. By this way, it is possible for the robots to depict and communicate the complex situations and their successions, as well as to learn the various complex skills and procedures through language.

Further, the thinking of robots requires the additional memory, inference and assumption, difficult for the zip function of computer. Whereas, because most thinking is either situational or successional, language is as well indispensable for assistance to the thinking of robots as stated above.

It is noted that, the words in robots, such as the noun, verb, adjective, adverb and so on, can be symbolized with both special characters and episodic associations [25], such as spatial, temporal, physical, biological and so on, helping to improve the comprehension and expression of the sentence more quickly than resorting to their individual visual, auditory, behavioral correspondents and so on, especially helpful to comprehend or express the central theme of long paragraph.

In all, with the semantic/syntactic/episodic components of language [1] [2], it is possible for the linguistic robots to acquire the advantages of human brain over animal brain, able to animate and depict the complex situations and processes, and to learn the various complex skills and procedures through language.

\section{Brief Perspectives}

The neural linguistic model of three semantic/syntactic/episodic components [1]

[2] can directly be installed into various forms of artificial intelligence and make them acquire the ability of language as humans. 
At the highest level, the robots of biological adaptive functions would certainly be installed with full language functions of the semantic/syntactic/episodic components [1] [2], which would greatly improve the performance of these linguistic robots, including the adaptive self-feeding robots, adaptive combating robots and tanks, some automated jet fighters, and so on.

At the middle level, the automated machines without biological adaptive functions may also be installed with the language functions to accomplish some specific tasks, which would also greatly improve the performance of such artificial intelligence, including the machine translation, novel animation, game characters, and so on.

At the lowest level, some artificial intelligence without biological adaptive functions may resort to some installed language functions for the convenience of manual operation of them, including the manually operated jet fighters, manually operated combating robots and tanks, manually operated researching robots, manual operation of machines and computers in business and industry, and so on.

On the other hand, there are still many problems ahead necessary to solve in future. 1) The new adaptive linguistic robots would acquire life as humans, so that there would raise many ethical and social problems. 2) The new adaptive linguistic robots may not necessarily have the arms, feet, ears, eyes and so on that humans have, nor the sexual drive, but may have other functional parts that humans do not have, such as the keyboard, DVD drive, wheels, radar, and so on, which means that there require more investigations on the new adaptive linguistic robots different from humans. 3) The adaptive linguistic robots also require education and training like humans, which also need further investigations.

\section{Conclusions}

In this article, it is first briefly introduced and reviewed the new neural linguistic model of three semantic/syntactic/episodic components proposed by Cai, encompassing the sentential meanings to the neural linguistic processes, and making it feasible to install the whole linguistic functions into robots through the three semantic/syntactic/episodic components. It is in further pointed out that language is the unique advantage of human brain over animal brain, especially advantageous to symbolize or summarize the complex situations, and delineate or depict their successions by paragraphs and stories from sentences. Although thinking requires the additional memory, inference and assumption difficult for the zip of computer, for the thinking mostly to be situational and successional, language is likewise indispensable as assistance to thinking. With the semantic/syntactic/episodic neurolinguistic components to install the full functions of language, it is possible for the artificial intelligence to acquire the advantage of linguistic human brain over the animal brain, improving the depiction and communication of complex situations and processes, as well as learning the various complex skills and procedures through language. 
Besides, word episodic symbolization can symbolize all of the special characteristics and episodic associations of a word and then store them in linguistic robots easily. Many nonverbal spatial and emotional information can be stored in association with words in robots, greatly increasing the ability of linguistic robots.

In all, the three semantic/syntactic/episodic linguistic components can greatly improve the artificial intelligence in such fields as adaptive self-feeding robots, adaptive combating robots and tanks, jet fighters, machine translation, novel animation, game characters, and so on.

\section{Acknowledgements}

It is herein acknowledged that Mingxun Cai paid the Open Access publication fee of this paper.

\section{Funding}

The author declares no financial support for this work.

\section{Conflict of Interest Statement}

The author declares no conflict of interest for this work.

\section{References}

[1] Cai, Z.-J. (2015) Semantic Memory Association, Procedural Grammar Syntax and Episodic Modality Coordination as Three Interactive Neural Processes Organizing Language: A Model. Open Access Library Journal, 2, e1718. https://doi.org/10.4236/oalib.1101718

[2] Cai, Z.-J. (2017) The Neurobiological Models of Language: An Updated Review. Psychology \& Neuroscience, 10, 297-306. https://doi.org/10.1037/pne0000101

[3] Pinker, S. (1991) Rules of Language. Science, 253, 530-535. https://doi.org/10.1126/science.1857983

[4] Ullman, M.T., Corkin, S., Coppola, M., Hickok, G., Growdon, J.H., Koroshetz, W.J. and Pinker, S. (1997) A Neural Dissociation within Language: Evidence That the Mental Dictionary Is Part of Declarative Memory, and That Grammatical Rules Are Processed by the Procedural System. Journal of Cognitive Neuroscience, 9, 266-276. https://doi.org/10.1162/jocn.1997.9.2.266

[5] Ullman, M.T. (2004) Contributions of Memory Circuits to Language: The Declarative/Procedural Model. Cognition, 92, 231-270. https://doi.org/10.1016/j.cognition.2003.10.008

[6] Cai, Z.-J. (1990) The Neural Mechanism of Declarative Memory Consolidation and Retrieval: A Hypothesis. Neuroscience \& Biobehavioral Reviews, 14, 295-304. https://doi.org/10.1016/S0149-7634(05)80039-9

[7] Cai, Z.-J. (2018) The Limbic-Reticular Coupling Theory of Memory Processing in the Brain and Its Greater Compatibility over Other Theories. Dementia e Neuropsychologia, 12, 105-113. https://doi.org/10.1590/1980-57642018dn12-020002

[8] Squire, L.R. (1987) Memory and Brain. Oxford University Press, Oxford.

[9] Maguire, G.A., Yu, B.P., Franklin, D.L. and Riley, G.D. (2004) Alleviating Stuttering 
with Pharmacological Interventions. Expert Opinion on Pharmacotherapy, 5, 1565-1571. https://doi.org/10.1517/14656566.5.7.1565

[10] Stager, S.V., Calis, K., Grothe, D., Bloch, M., Berensen, N.M., Smith, P.J. and Braun, A. (2005) Treatment with Medications Affecting Dopaminergic and Serotonergic Mechanisms: Effects on Fluency and Anxiety in Persons Who Stutter. Journal of Fluency Disorders, 30, 319-335. https://doi.org/10.1016/j.jfludis.2005.09.004

[11] Lan, J., Song, M., Pan, C., Zhuang, G., Wang, Y., Ma, W., Chu, Q., Lai, Q., Xu, F., Li, Y., Liu, L. and Wang, W. (2009) Association between Dopaminergic Genes (SLC6A3 and DRD2) and Stuttering among Han Chinese. Journal of Human Genetics, 54, 457-460. https://doi.org/10.1038/jhg.2009.60

[12] Small, S.L. and Llano, D.A. (2009) Biological Approaches to Aphasia Treatment. Current Neurology and Neuroscience Reports, 9, 443-450.

https://doi.org/10.1007/s11910-009-0066-x

[13] Berthier, M.L., Pulvermüller, F., Dávila, G., Casares, N.G. and Gutiérrez, A. (2011) Drug Therapy of Post-Stroke Aphasia: A Review of Current Evidence. Neuropsychology Review, 21, 302-317. https://doi.org/10.1007/s11065-011-9177-7

[14] Cape, E.G., Manns, I.D., Alonso, A., Beaudet, A. and Jones, B.E. (2000) Neurotensin-Induced Bursting of Cholinergic Basal Forebrain Neurons Promotes Gamma and Theta Cortical Activity together with Waking and Paradoxical Sleep. The Journal of Neuroscience, 20, 8452-8461. https://doi.org/10.1523/JNEUROSCI.20-22-08452.2000

[15] Mena-Segovia, J., Sims, H.M., Magill, P.J. and Bolam, J.P. (2008) Cholinergic Brainstem Neurons Modulate Cortical Gamma Activity during Slow Oscillations. The Journal of Physiology, 586, 2947-2960. https://doi.org/10.1113/jphysiol.2008.153874

[16] Wang, L., Zhu, Z. and Bastiaansen, M. (2012) Integration or Predictability? A Further Specification of the Functional Role of Gamma Oscillations in Language Comprehension. Frontiers in Psychology, 3, 187. https://doi.org/10.3389/fpsyg.2012.00187

[17] Vidal, J.R., Freyermuth, S., Jerbi, K., Hamamé, C.M., Ossandon, T., Bertrand, O., Minotti, L., Kahane, P., Berthoz, A. and Lachaux, J.P. (2012) Long-Distance Amplitude Correlations in the High $\gamma$ Band Reveal Segregation and Integration within the Reading Network. The Journal of Neuroscience, 32, 6421-6434.

https://doi.org/10.1523/JNEUROSCI.4363-11.2012

[18] Weiss, S. and Müller, H.M. (2013) The Non-Stop Road from Concrete to Abstract: High Concreteness Causes the Activation of Long-Range Networks. Frontier in Human Neuroscience, 7, 526. https://doi.org/10.3389/fnhum.2013.00526

[19] Miniscalco, C., Hagberg, B., Kadesjö, B., Westerlund, M. and Gillberg, C. (2007) Narrative Skills, Cognitive Profiles and Neuropsychiatric Disorders in 7-8-Year-Old Children with Late Developing Language. International Journal of Language and Communication Disorders, 42, 665-681. https://doi.org/10.1080/13682820601084428

[20] Ash, S., Menaged, A., Olm, C., McMillan, C.T., Boller, A., Irwin, D.J., McCluskey, L., Elman, L. and Grossman, M. (2014) Narrative Discourse Deficits in Amyotrophic Lateral Sclerosis. Neurology, 83, 520-528. https://doi.org/10.1212/WNL.0000000000000670

[21] Youse, K.M. and Coelho, C.A. (2009) Treating Underlying Attention Deficits as a Means for Improving Conversational Discourse in Individuals with Closed Head Injury: A Preliminary Study. NeuroRehabilitation, 24, 355-364. 
[22] DiLollo, A., Neimeyer, R.A. and Manning, W.H. (2002) A Personal Construct Psychology View of Relapse: Indications for a Narrative Therapy Component to Stuttering Treatment. Journal of Fluency Disorders, 27, 19-40. https://doi.org/10.1016/S0094-730X(01)00109-7

[23] Leahy, M.M., O’Dwyer, M. and Ryan, F. (2012) Witnessing Stories: Definitional Ceremonies in Narrative Therapy with Adults Who Stutter. Journal of Fluency Disorders, 37, 234-241. https://doi.org/10.1016/j.jfludis.2012.03.001

[24] Cai, Z.-J. (2016) Principles Derived from Neurolinguistics of Brain for Design of Translation Machines. Open Access Library Journal, 3, e2704. https://doi.org/10.4236/oalib.1102704

[25] Cai, Z.-J. (2017) Three Types of Episodic Associations for the Semantic/Syntactic/Episodic Model of Language Prospective in Applications to the Statistical Translation. Open Access Library Journal, 4, e3830. https://doi.org/10.4236/oalib.1103830

[26] Cai, Z.-J. (1991) The Functions of Sleep: Further Analysis. Physiology \& Behavior, 50, 53-60. https://doi.org/10.1016/0031-9384(91)90497-C

[27] Cai, Z.-J. (2016) Progressions of Sleep, Memory and Depression Applicable to Psychoanalysis: A Review. Current Psychiatry Reviews, 12, 240-245. https://doi.org/10.2174/1573400512666160610083505

[28] Friederici, A.D. (2011) The Brain Basis of Language Processing: From Structure to Function. Physiological Reviews, 91, 1357-1392.

https://doi.org/10.1152/physrev.00006.2011

[29] Somers, M., Shields, L.S., Boks, M.P., Kahn, R.S. and Sommer, I.E. (2015) Cognitive Benefits of Right-Handedness: A Meta-Analysis. Neuroscience \& Biobehavioral Reviews, 51, 48-63. https://doi.org/10.1016/j.neubiorev.2015.01.003 